Review began 02/09/2022 Review ended 02/17/2022 Published 02/22/2022

๑) Copyright 2022

Fujikawa et al. This is an open access article distributed under the terms of the Creative Commons Attribution License CCBY 4.0., which permits unrestricted use, distribution, and reproduction in any medium, provided the original author and source are credited.

\section{Early Postoperative Rehabilitation Using the Hybrid Assistive Limb (HAL) Lumbar Type in Patients With Hip Fracture: A Pilot Study}

Tomohiro Fujikawa ${ }^{1}$, Seita Takahashi ${ }^{1}$, Naoki Shinohara ${ }^{2}$, Naohiko Mashima ${ }^{3}$, Masao Koda ${ }^{4}$, Hiroshi Takahashi $^{4}$, Yoshihiro Yasunaga ${ }^{4}$, Yoshiyuki Sankai ${ }^{5}$, Masashi Yamazaki ${ }^{4}$, Kousei Miura ${ }^{4}$

1. Department of Rehabilitation, HITO Medical Center, Shikokuchuo, JPN 2. Department of Orthopaedics, HITO Medical Center, Shikokuchuo, JPN 3. Department of Regeneration of Community Medicine, Ehime University Graduate School of Medicine, Toon-shi, JPN 4. Department of Orthopaedic Surgery, Faculty of Medicine, University of Tsukuba, Tsukuba, JPN 5. Center for Cybernics Research, University of Tsukuba, Tsukuba, JPN

Corresponding author: Kousei Miura,kmiura@tsukuba-seikei.jp

\section{Abstract}

Objective: To extend life expectancy after surgery, patients with hip fractures need to improve their mobility quickly through postoperative rehabilitation. Voluntary hip joint motion supported by the hybrid assistive limb (HAL) lumbar type, an exoskeleton robot suit characterized by its ability to detect the wearer's intentions through the bioelectrical signals and assist hip extension motions at an optimal timing, may be effective to improve mobility in patients with hip joint dysfunction after surgery. We aimed to introduce rehabilitation using the HAL lumbar type in the early period after hip fracture surgery.

Methods: Patients who underwent internal fixation for hip fracture at a single institution were prospectively enrolled. They received early postoperative rehabilitation (forward and backward bending of the lumbar spine, pelvic tilt forward and backward, standing up, and squatting) using the HAL lumbar type (six times a week for 15 min per session). Five-times-sit-to-stand (FTSS) and timed-up-and-go (TUG) tests were conducted at baseline before HAL rehabilitation (pre-HAL) and after the HAL rehabilitation (post-HAL) intervention.

Results: We enrolled 14 patients (one man, 13 women) in this study. There were no adverse events, and all patients were able to complete the entire rehabilitation program. Post-HAL FTSS showed significant improvement compared with pre-HAL and had a large effect size of 1.81 (95\% CI $=0.93$ to 2.66 ) and sufficient power.

Conclusions: Robotic rehabilitation with HAL lumbar type could be introduced without adverse events, even in the early postoperative period following surgery for hip fracture. Further study is needed to develop an appropriate rehabilitation protocol using the HAL lumbar type.

Categories: Orthopedics, Trauma

Keywords: exoskeleton, robot rehabilitation, postoperative rehabilitation, hybrid assistive limb, hip fracture

\section{Introduction}

Aging societies have led to increased hip fractures among the elderly worldwide [1]. Hip fracture significantly reduces physical function and quality of life even after surgery [2,3]. Furthermore, the hip fracture has been reported to be a threat to life expectancy [4]. In particular, delayed walking after hip fracture surgery has been associated with poor life expectancy [5]. Therefore, patients who undergo surgery for hip fracture need to improve their mobility quickly through postoperative rehabilitation.

Lee et al. [6] found early mobilization is strongly recommended for rehabilitation after hip fracture surgery. However, rehabilitation procedures after hip fracture surgery have not yet been fully established [7]. Robotic rehabilitation has been used in the postoperative rehabilitation of various diseases. The hybrid assistive limb (HAL; Cyberdyne Inc., Ibaraki, Japan) can estimate the wearer's motion intention through bioelectrical signals (BES) and provide coordinated joint motion support with appropriate timing and torque. Three types of HAL (lower limb type, single-joint type, lumbar type) are currently being attempted for rehabilitation. There are a number of studies of postoperative rehabilitation using the HAL lower limb type for myelopathy [8,9], cerebral palsy [10], and knee osteoarthritis [11,12]. Rehabilitation using the HAL single-joint type after surgery for knee osteoarthritis has also been reported [13,14]. In this study, we focused on the HAL lumbar type. The HAL lumbar type can detect BES of the lumbar erector spinae muscle and support the wearer's hip extension movement at optimal timing [15]. It was initially developed as a device to reduce the lumbar load during heavy work and has been reported to reduce the lumbar load during lifting [16], snow-shoveling [17], and simulated patient transfer movements [18]. More recently, it has been considered that support for hip extension movements by the HAL lumbar type may also be effective in supporting the standing movement. 


\section{Cureus}

To date, robotic rehabilitation using the HAL lumbar type has been attempted for locomotive syndrome [19] and frailty [20]. However, few studies have focused on postoperative rehabilitation in hip fracture patients using the HAL lumbar type. Hip joint motion support by the HAL lumbar type may be effective for hip fracture patients because they have hip joint dysfunction after surgery. This study aimed to introduce rehabilitation using the HAL lumbar type for patients in the early postoperative period after hip fracture surgery.

\section{Materials And Methods}

\section{Participants}

This study is a pilot, prospective, single-arm, inpatient postoperative rehabilitation study using the HAL lumbar type for patients following hip fracture surgery. Patients who underwent internal fixation for hip fracture at a single institution between April 2019 and June 2020 and satisfied the following inclusion criteria were enrolled: (1) able to walk independently or with a cane before hip fracture; (2) immediate full weight-bearing after surgery was allowed. We excluded the patients who met the following criteria: (1) severe cognitive impairment that makes it difficult to understand the rehabilitation protocol; (2) difficult to walk due to cerebrovascular disease before surgery; (3) implanted pacemakers; (4) underwent bipolar hip arthroplasty. We obtained written informed consent from all participants. This study was conducted following the Declaration of Helsinki with the approval of the Ethical Review Committee of HITO Hospital (Approval No.: 20200107002).

\section{Early postoperative rehabilitation with the HAL lumbar type}

The HAL lumbar type consists of a lumbar frame, thigh mold power units, and electrode detecting BES (Figure 1). 


\section{Cureus}

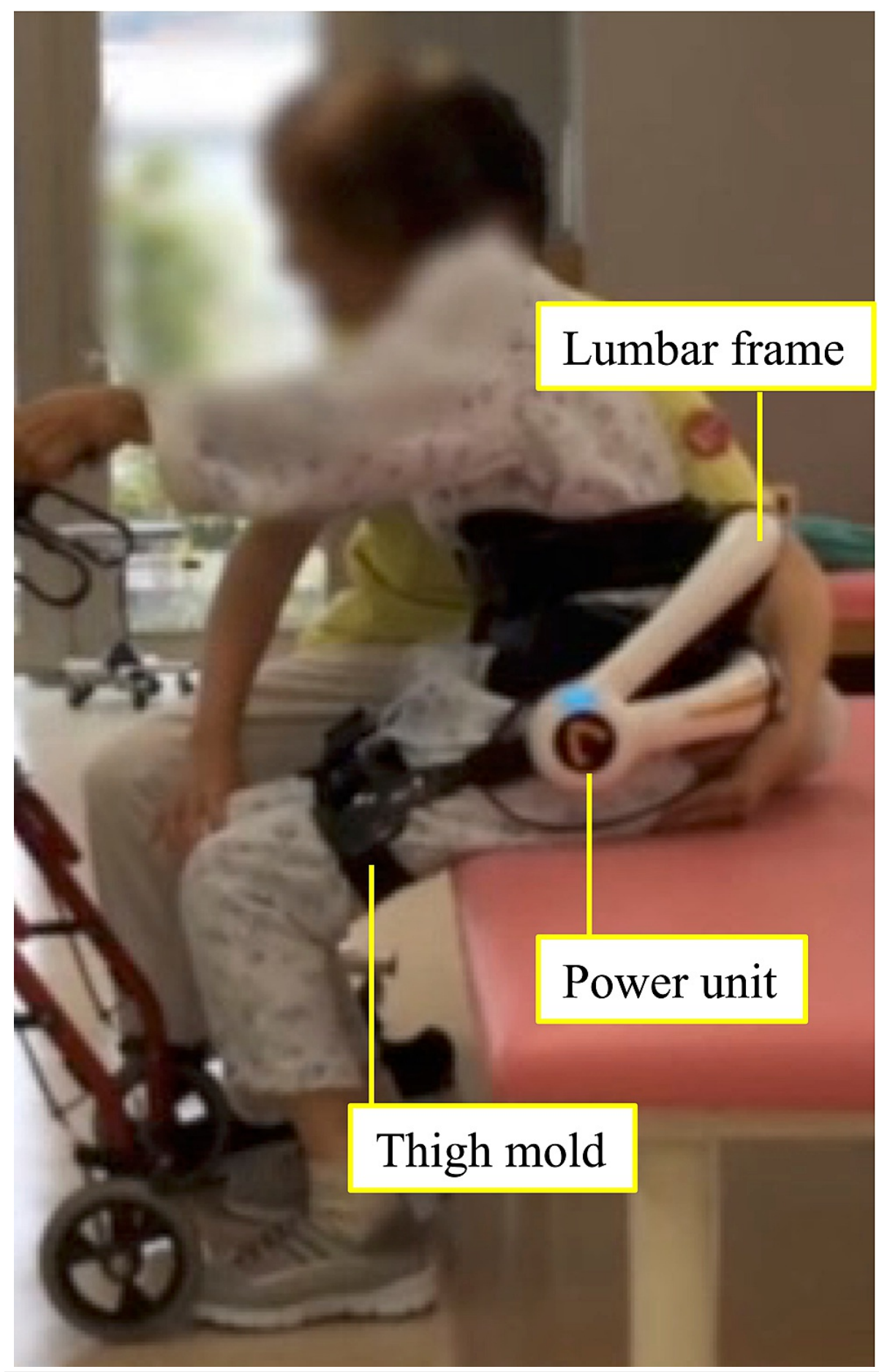

\section{FIGURE 1: Hybrid assistive limb (HAL) lumbar type}

The electric motors in the power units can assist hip joint extension motion when a wearer stands up.

It is equipped with angle sensors in the power units located in the bilateral hip joint to detect the wearer's hip joint angle. The electric motors in the power units can support hip joint extension when the wearer stands up. Based on BES detected from electrodes attached to the lumbar erector spinae muscles, the HAL lumbar type has the characteristic of providing hip joint motion support at the timing intended by the wearer [15].

Participants underwent rehabilitation using the HAL lumbar type in addition to the usual conventional postoperative rehabilitation after hip fracture surgery. Once the patient's general condition was stabilized after surgery, HAL rehabilitation was started as soon as possible. The following four types of HAL rehabilitation were performed: (1) forward and backward bending of the lumbar spine in a sitting position; 


\section{Cureus}

(2) pelvic tilt forward and backward in a sitting position; (3) standing up from a sitting position with the support of the upper limbs (Figure 2); (4) squatting in a standing position with the support of the upper limbs (Figure 3).

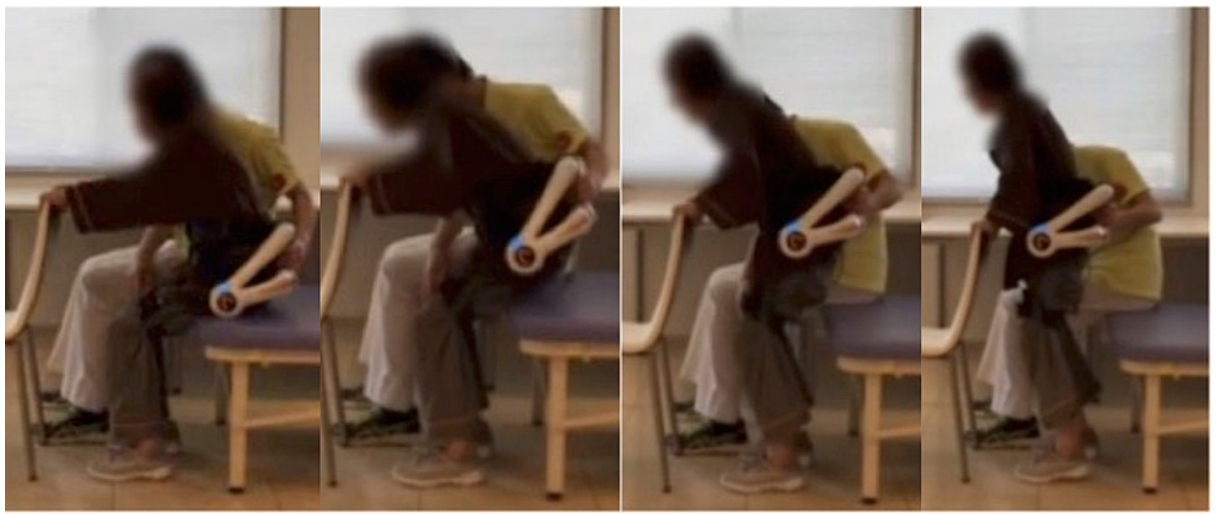

FIGURE 2: Standing up exercise from a sitting position

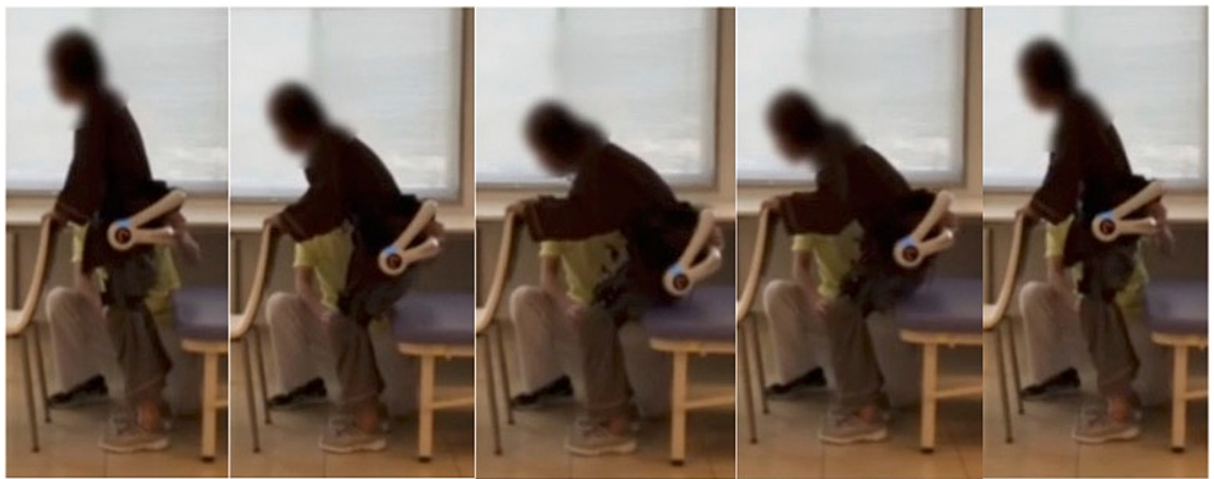

FIGURE 3: Squatting exercise in a standing position

The HAL rehabilitation was conducted six times a week for 15 minutes per session. Rehabilitation using the HAL lumbar type was completed when the patient became able to walk with a walker.

\section{Outcome measures}

The five-times-sit-to-stand (FTSS) and the timed-up-and-go (TUG) tests were used to assess locomotion function. These tests were conducted at baseline before HAL rehabilitation (pre-HAL) and after the HAL rehabilitation intervention (post-HAL). The FTSS test was performed at maximum speed while holding onto a 40 -cm high platform. The TUG test was performed using a walker both pre-HAL and post-HAL.

A Wilcoxon signed-rank test was used to compare pre-HAL and post-HAL test results. Statistical analysis was performed using the JMP software package version 14.0.0 (SAS Institute, Cary, NC, USA). Effect size and post-hoc statistical power were calculated using Gpower software (version 3.1.9, University of Dusseldorf, Dusseldorf, Germany). P $<0.05$ was considered significant.

\section{Results}

Fourteen patients (one man, 13 women) were enrolled in this study. No patient met pre-set exclusion criteria. The demographic data of the participants are summarized in Table 1. 


\section{Cureus}

\begin{tabular}{|c|c|c|}
\hline Characteristics & $\square$ & $\square$ \\
\hline Sample size & $\mathrm{N}(\%)$ & 14 \\
\hline Age (years) & Mean \pm SD (range) & $83.4 \pm 8.4(69-96)$ \\
\hline $\operatorname{Sex}(\%)$ & Male & $1(7.1)$ \\
\hline$\square$ & Female & $13(92.9)$ \\
\hline Fracture type (\%) & $\mathrm{FN}$ & $2(14.3)$ \\
\hline$\square$ & FT & $12(85.7)$ \\
\hline Ambulation before HF (\%) & Independent & $13(92.9)$ \\
\hline$\square$ & Cane & $1(7.1)$ \\
\hline HAL start (POD) & Mean \pm SD (range) & $5.9 \pm 2.5(2-8)$ \\
\hline
\end{tabular}

\section{TABLE 1: Patient demographic data}

$\mathrm{FN}$, femoral neck fracture; FT, femoral trochanteric fracture; HF, hip fracture; HAL, hybrid assistive limb; POD, postoperative day

There were no adverse events associated with rehabilitation using the HAL lumbar type, and all participants were able to complete the entire rehabilitation program. The clinical characteristics of each case are summarized in Table 2 . 


\begin{tabular}{|c|c|c|c|c|c|c|c|c|c|c|c|c|}
\hline Case & $\begin{array}{l}\text { Age } \\
\text { (years) }\end{array}$ & Sex & $\begin{array}{l}\text { Fracture } \\
\text { type }\end{array}$ & $\begin{array}{l}\text { Ambulation } \\
\text { before HF }\end{array}$ & $\begin{array}{l}\text { HAL start } \\
\text { (POD) }\end{array}$ & $\begin{array}{l}\text { Walker start } \\
\text { (POD) }\end{array}$ & $\square$ & $\begin{array}{l}\text { Pre-HAL } \\
\text { FTSS }\end{array}$ & $\begin{array}{l}\text { POSt-HAL } \\
\text { FTSS }\end{array}$ & $\square$ & $\begin{array}{l}\text { Pre- } \\
\text { HAL } \\
\text { TUG }\end{array}$ & $\begin{array}{l}\text { Post- } \\
\text { HAL } \\
\text { TUG }\end{array}$ \\
\hline 1 & 72 & $\mathrm{~F}$ & $\mathrm{FN}$ & Independent & 8 & 11 & $\square$ & 16.4 & 8.22 & $\square$ & 15.2 & 8.2 \\
\hline 2 & 85 & $\mathrm{~F}$ & FN & Independent & 8 & 16 & $\square$ & 35.5 & 31.4 & $\square$ & 48.7 & 25.4 \\
\hline 3 & 85 & $\mathrm{~F}$ & FT & Independent & 8 & 13 & $\square$ & 41.3 & 12.33 & $\square$ & 101.2 & 18.9 \\
\hline 4 & 70 & $\mathrm{~F}$ & FT & Independent & 8 & 11 & $\square$ & 32.7 & 18.2 & $\square$ & 16.3 & 11.1 \\
\hline 5 & 91 & $\mathrm{~F}$ & $\mathrm{FT}$ & Independent & 8 & 14 & $\square$ & 42.2 & 15.1 & $\square$ & - & 21.4 \\
\hline 6 & 96 & $\mathrm{~F}$ & FT & Independent & 8 & 19 & $\square$ & 43.2 & 22.6 & $\square$ & - & 13.7 \\
\hline 7 & 80 & $\mathrm{~F}$ & $\mathrm{FT}$ & Independent & 8 & 12 & $\square$ & 41.2 & 21.32 & $\square$ & 72.0 & 19.4 \\
\hline 8 & 84 & $\mathrm{~F}$ & $\mathrm{FT}$ & Independent & 8 & 10 & $\square$ & 26.4 & 20.22 & $\square$ & 59.7 & 32.4 \\
\hline 9 & 69 & $M$ & FT & Independent & 3 & 7 & $\square$ & 29.2 & 6.99 & $\square$ & - & 14.6 \\
\hline 10 & 83 & $\mathrm{~F}$ & FT & Independent & 4 & 22 & $\square$ & 39.9 & 27.84 & $\square$ & - & 22.9 \\
\hline 11 & 90 & $\mathrm{~F}$ & $\mathrm{FT}$ & Independent & 4 & 12 & $\square$ & 32.4 & 15.67 & $\square$ & - & 53.4 \\
\hline 12 & 87 & $\mathrm{~F}$ & FT & Independent & 2 & 7 & $\square$ & 45.3 & 25.34 & $\square$ & - & 24.8 \\
\hline 13 & 94 & $\mathrm{~F}$ & $\mathrm{FT}$ & Cane & 3 & 8 & $\square$ & 38.4 & 33.72 & $\square$ & 32.9 & 29.7 \\
\hline 14 & 81 & $\mathrm{~F}$ & $\mathrm{FT}$ & Independent & 3 & 10 & $\square$ & 24.5 & 18.24 & $\square$ & - & 21.6 \\
\hline $\begin{array}{l}\text { Mean } \\
\pm \text { SD }\end{array}$ & $\square$ & $\square$ & $\square$ & $\square$ & $\square$ & $\square$ & $\square$ & $\begin{array}{l}34.9 \pm \\
8.4\end{array}$ & $19.8 \pm 8.0^{*}$ & $\square$ & $\square$ & 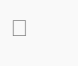 \\
\hline $\begin{array}{l}\text { Effect } \\
\text { size }\end{array}$ & $\square$ & $\square$ & $\square$ & $\square$ & $\square$ & $\square$ & $\square$ & \multicolumn{2}{|c|}{$\begin{array}{l}1.81(95 \% \mathrm{Cl}=0.93- \\
2.66)\end{array}$} & $\square$ & $\square$ & $\square$ \\
\hline Power & $\square$ & $\square$ & $\square$ & $\square$ & $\square$ & $\square$ & $\square$ & \multicolumn{2}{|l|}{0.99} & $\square$ & $\square$ & $\square$ \\
\hline
\end{tabular}

\section{TABLE 2: Patient clinical characteristics}

Values are mean \pm standard deviation. FN, femoral neck fracture; FT, femoral trochanteric fracture; HF, hip fracture; HAL, hybrid assistive limb; POD, postoperative day; FTSS, five-times-sit-to-stand test; TUG, timed-up-and-go test; ${ }^{*} P<0.01$ (compared with Pre-HAL FTSS)

Post-HAL FTSS showed significant improvement compared to pre-HAL with a large effect size of 1.81 (95\% $\mathrm{CI}=0.93$ to 2.66 ) and sufficient power (0.99). In pre-HAL tests, seven out of 14 patients could not walk sufficiently to complete the TUG test, but all patients could achieve it in post-HAL. In all other cases, the TUG time decreased.

\section{Discussion}

In the present study, we aimed to introduce rehabilitation using the HAL lumbar type in the early postoperative period following surgery for a hip fracture. All 14 patients were able to complete the HAL rehabilitation without adverse events. Furthermore, post-HAL FTSS test results showed improvement compared to pre-HAL.

Various rehabilitation procedures after hip fracture surgery have been reported. In a review by Lee et al. [21], they found balance training to positively affect balance, gait, leg strength, and activity in daily life (ADL) after hip fracture surgery. Progressive resistance exercise also has a positive effect on mobility [22]. Early maximum strength training after hip fracture surgery is effective in improving leg strength [23]. By contrast, according to a review by Hulsbæk et al. [24], exercise therapy after hip fracture is not sufficiently effective. Lee et al. reported that physical therapy during the postoperative acute care phase is essential for early discharge, but it is not possible in all cases [6]. That is because fractures, inflammatory agents, and pain associated with surgical invasion occur in the perioperative period following hip fracture surgery and are known to lead to lost opportunities for rehabilitation interventions $[25,26]$. Therefore, it seems essential to determine a way to assist early rehabilitation intervention after hip fracture surgery.

In the present study, we attempted early postoperative rehabilitation intervention using the HAL lumbar 
type. To date, several reports have shown that exercise therapy using the HAL lumbar type improved physical functions in the elderly. Kotani et al. [20] reported that five core and squat exercise sessions with the HAL lumbar type for frailty improved motor function, including TUG times. Miura et al. [19] found that balance function improved after 12 sessions of exercise therapy with the HAL lumbar type consisting of sitto-stand, lumbar flexion-extension, and gait training for the locomotive syndrome. Both studies found that exercise therapy using the HAL lumbar type could be safely performed by elderly patients without exacerbation of pain. A case of exercise therapy for postoperative rehabilitation using HAL lumbar type after lumbar fusion surgery has been reported [27]. To our knowledge, this is the first report of robotic rehabilitation after hip fracture surgery. This study preliminarily introduced the following four types of rehabilitation using the HAL lumbar type: forward and backward bending of the lumbar spine, pelvic tilt forward and backward, standing up and squatting. These exercises appear to increase hip joint function and improve stride length, resulting in increased walking speed. The HAL lumbar type is characterized by its ability to detect the wearer's intentions through BES and assist hip extension motions at optimal timing. In addition, the motor learning effect induced by interactive biofeedback can provide coordinated joint motion support, which may result in the prevention of overload [28]. Hip fracture occurs mainly in the elderly, so frailty and early postoperative pain may inhibit rehabilitation. For those factors, rehabilitation of hip motion using the HAL lumbar type may be more effective than conventional rehabilitation.

Several limitations of the present study should be considered. The effect of the HAL lumbar type on physical function could not be clarified because there was no comparison with the control group, which received only conventional rehabilitation. It was impossible to prove that the HAL lumbar type reduced the physical load because there was no evaluation of pain and EMG data changes during rehabilitation. Future comparative studies with large sample sizes will be needed to resolve these limitations.

\section{Conclusions}

Robotic rehabilitation with HAL lumbar type could be introduced without adverse events, even in the early postoperative period following internal fixation for hip fracture. Post-HAL FTSS test results showed improvement compared to pre-HAL FTSS test results. These findings suggest that HAL lumbar type may be an option for early postoperative rehabilitation after hip fracture surgery. Further study is needed to demonstrate the effect of HAL lumbar type on physical function and to develop an appropriate rehabilitation protocol.

\section{Additional Information \\ Disclosures}

Human subjects: Consent was obtained or waived by all participants in this study. Ethical Review Committee of HITO Hospital issued approval 20200107002. We obtained documented informed consent from all participants. Animal subjects: All authors have confirmed that this study did not involve animal subjects or tissue. Conflicts of interest: In compliance with the ICMJE uniform disclosure form, all authors declare the following: Payment/services info: All authors have declared that no financial support was received from any organization for the submitted work. Financial relationships: Yoshiyuki Sankai and Yoshihiro Yasunaga declare(s) employment from Cyberdyne, Inc. A commercial party having a direct financial interest in the results of the research supporting this article has conferred or will confer a financial benefit to one or more of the authors. Yoshihiro Yasunaga is a Ph.D. student at the University of Tsukuba and the general manager of sales and director of the university's venture company “Cyberdyne, Inc.” in Ibaraki, Japan. Yoshiyuki Sankai is an inventor of the world's first wearable cyborg HAL, a professor at the University of Tsukuba, a founder, a shareholder, and the CEO of the university's venture company Cyberdyne. Conflicts of interest are strictly managed by the University of Tsukuba according to national university rules and guidelines and by the board of directors of Cyberdyne. Patents of HAL belongs to the University of Tsukuba. Patent royalties are paid to the University of Tsukuba from Cyberdyne, and part of the royalties are paid to Yoshiyuki Sankai from the University of Tsukuba according to national university rules. Cyberdyne is a R\&D company and the manufacturer of the HAL. The present report was proposed by the authors. Cyberdyne was not directly involved in the study design; collection, analysis, or interpretation of data; writing of the report; or the decision to submit the paper for publication. No commercial party having a direct financial interest in the results of the research supporting this article has or will confer a benefit to the authors or any organization with which the other authors are associated (Tomohiro Fujikawa, Seita Takahashi, Naoki Shinohara, Naohiko Mashima, Masao Koda, Hiroshi Takahashi, Masashi Yamazaki and Kousei Miura). Other relationships: All authors have declared that there are no other relationships or activities that could appear to have influenced the submitted work.

\section{References}

1. Veronese N, Maggi S: Epidemiology and social costs of hip fracture . Injury. 2018, 49:1458-60. 10.1016/j.injury.2018.04.015

2. Alexiou KI, Roushias A, Varitimidis SE, Malizos KN: Quality of life and psychological consequences in elderly patients after a hip fracture: a review. Clin Interv Aging. 2018, 13:143-50. 10.2147/CIA.S150067

3. Peeters CM, Visser E, Van de Ree CL, Gosens T, Den Oudsten BL, De Vries J: Quality of life after hip fracture in the elderly: a systematic literature review. Injury. 2016, 47:1369-82. 10.1016/j.injury.2016.04.018 
4. Valizadeh M, Mazloomzadeh S, Golmohammadi S, Larijani B: Mortality after low trauma hip fracture: a prospective cohort study. BMC Musculoskelet Disord. 2012, 13:143. 10.1186/1471-2474-13-143

5. Siu AL, Penrod JD, Boockvar KS, Koval K, Strauss E, Morrison RS: Early ambulation after hip fracture: effects on function and mortality. Arch Intern Med. 2006, 166:766-71. 10.1001/archinte.166.7.766

6. Lee KJ, Um SH, Kim YH: Postoperative rehabilitation after hip fracture: a literature review . Hip Pelvis. 2020, 32:125-31. 10.5371/hp.2020.32.3.125

7. Falaschi P, Marsh D: Orthogeriatrics: the management of older patients with fragility fractures . Springer, Cham, CH; 2021. 10.1007/978-3-030-48126-1

8. Kubota S, Abe T, Kadone H, et al.: Hybrid assistive limb (HAL) treatment for patients with severe thoracic myelopathy due to ossification of the posterior longitudinal ligament (OPLL) in the postoperative acute/subacute phase: a clinical trial. J Spinal Cord Med. 2019, 42:517-25. 10.1080/10790268.2018.1525975

9. Yatsugi A, Morishita T, Fukuda H, et al.: Feasibility of neurorehabilitation using a hybrid assistive limb for patients who underwent sine surgery. Appl Bionics Biomech. 2018, 2018:7435746. 10.1155/2018/7435746

10. Mataki Y, Kamada H, Mutsuzaki H, et al.: Use of hybrid assistive limb (HAL ${ }^{\circledR}$ ) for a postoperative patient with cerebral palsy: a case report. BMC Res Notes. 2018, 11:201. 10.1186/s13104-018-3311-z

11. Tanaka Y, Oka H, Nakayama S, et al.: Improvement of walking ability during postoperative rehabilitation with the hybrid assistive limb after total knee arthroplasty: a randomized controlled study. SAGE Open Med. 2017, 5:2050312117712888. 10.1177/2050312117712888

12. Yoshikawa K, Mutsuzaki H, Sano A, Koseki K, Fukaya T, Mizukami M, Yamazaki M: Training with hybrid assistive limb for walking function after total knee arthroplasty. J Orthop Surg Res. 2018, 13:163. 10.1186/s13018-018-0875-1

13. Goto K, Morishita T, Kamada S, et al.: Feasibility of rehabilitation using the single-joint hybrid assistive limb to facilitate early recovery following total knee arthroplasty: a pilot study. Assist Technol. 2017, 29:197-201. 10.1080/10400435.2016.1219883

14. Yoshioka T, Kubota S, Sugaya H, Arai N, Hyodo K, Kanamori A, Yamazaki M: Feasibility and efficacy of knee extension training using a single-joint hybrid assistive limb, versus conventional rehabilitation during the early postoperative period after total knee arthroplasty. J Rural Med. 2021, 16:22-8. 10.2185/jrm.2020-024

15. Hara H, Sankai Y: Development of HAL for lumbar support . J- STAGE. 2010, 2010:416-21. 10.14864/softscis.2010.0.416.0

16. Miura K, Kadone H, Koda M, et al.: The hybrid assistive limb (HAL) for Care Support successfully reduced lumbar load in repetitive lifting movements. J Clin Neurosci. 2018, 53:276-9. 10.1016/j.jocn.2018.04.057

17. Miura K, Kadone H, Koda M, et al.: The hybrid assisted limb (HAL) for care support, a motion assisting robot providing exoskeletal lumbar support, can potentially reduce lumbar load in repetitive snow-shoveling movements. J Clin Neurosci. 2018, 49:83-6. 10.1016/j.jocn.2017.11.020

18. Miura K, Kadone H, Abe T, et al.: Successful use of the hybrid assistive limb for care support to reduce lumbar load in a simulated patient transfer. Asian Spine J. 2021, 15:40-5. 10.31616/asj.2019.0111

19. Miura K, Koda M, Tamaki K, et al.: Exercise training using hybrid assistive limb (HAL) lumbar type for locomotive syndrome: a pilot study. BMC Musculoskelet Disord. 2021, 22:533. 10.1186/s12891-021-04421-3

20. Kotani N, Morishita T, Yatsugi A, et al.: Biofeedback Core Exercise Using Hybrid Assistive Limb for Physical Frailty Patients With or Without Parkinson's Disease. Front Neurol. 2020, 11:215. 10.3389/fneur.2020.00215

21. Lee SY, Jung SH, Lee SU, Ha YC, Lim JY: Effect of balance training after hip fracture surgery: a systematic review and meta-analysis of randomized controlled sudies. J Gerontol A Biol Sci Med Sci. 2019, 74:1679-85. 10.1093/gerona/gly271

22. Lee SY, Yoon BH, Beom J, Ha YC, Lim JY: Effect of lower-limb progressive resistance exercise after hip fracture surgery: a systematic review and meta-analysis of randomized controlled studies. J Am Med Dir Assoc. 2017, 18:1096.e19-26. 10.1016/j.jamda.2017.08.021

23. Berg OK, Stutzer JM, Hoff J, Wang E: Early maximal strength training improves leg strength and postural stability in elderly following hip fracture surgery. Geriatr Orthop Surg Rehabil. 2021, 12:21514593211015103. 10.1177/21514593211015103

24. Hulsbæk S, Juhl C, Røpke A, Bandholm T, Kristensen MT: Exercise therapy is effective at improving shortand long-term mobility, ADL and balance in older patients following hip fracture: a systematic review and meta-analysis [PREPRINT]. J Gerontol A Biol Sci Med Sci. 2021, 10.1093/gerona/glab236

25. Elboim-Gabyzon M, Andrawus Najjar S, Shtarker H: Effects of transcutaneous electrical nerve stimulation (TENS) on acute postoperative pain intensity and mobility after hip fracture: a double-blinded, randomized trial. Clin Interv Aging. 2019, 14:1841-50. 10.2147/CIA.S203658

26. Morrison SR, Magaziner J, McLaughlin MA, et al.: The impact of post-operative pain on outcomes following hip fracture. Pain. 2003, 103:303-11. 10.1016/S0304-3959(02)00458-X

27. Yasunaga Y, Miura K, Koda M, et al.: Exercise therapy using the lumbar-type hybrid assistive limb ameliorates locomotive function after lumbar fusion surgery in an elderly patient. Case Rep Orthop. 2021, 2021:1996509. 10.1155/2021/1996509

28. Morishita T, Inoue T: Interactive bio-feedback therapy using hybrid assistive limbs for motor recovery after stroke: current practice and future perspectives. Neurol Med Chir (Tokyo). 2016, 56:605-12. 10.2176/nmc.st.2016-0094 